\title{
Comparison of Glucocorticoid and Nonsteroidal Anti-Inflammatory Drug Requirement Before and After Tumor Necrosis Factor Inhibitor Treatment in Patients With Rheumatoid Arthritis
}

\author{
Merih BİRLİK, ${ }^{1}$ Sedat ÇAPAR, ${ }^{2}$ Servet AKAR, ${ }^{1}$ İsmail SARI, ${ }^{1}$ Fatoş ÖNEN, ${ }^{1}$ Nurullah AKKOÇ \\ ${ }^{1}$ Department of Rheumatology, Medical Faculty of Dokuz Eylül University, İmir, Turkey \\ ${ }^{2}$ Department of Statistics, Dokuz Eylul University, Faculty of Science, İzmir, Turkey
}

\begin{abstract}
Objectives: This study aims to investigate whether use of nonsteroidal anti-inflammatory drugs (NSAIDs) or glucocorticoids (GCs) decreases over different time intervals before and after anti-tumor necrosis factor (anti-TNF) therapy in rheumatoid arthritis (RA) patients.

Patients and methods: Data were extracted from electronic prescription records of the Pension Fund of Turkey covering records from the years 2000 to 2005 to detect patients aged 18 years or older who were administered anti-TNF agents. All TNF blockers, NSAIDs and GCs which were available in the market were our target of investigation. Patients were categorized into three groups defined by duration of continuous anti-TNF use (episode-1: $>90$ days, episode-2: $>180$ days, episode-3: $>365$ days). First anti-TNF prescription detected in the database was identified as the index prescription and its date was taken as the start date of therapy. GC and NSAID consumptions during the first three, six and 12 months before initiation of anti-TNF agents were compared with the subsequent same duration of consumptions after the initiation of anti-TNF agents.

Results: Comparison of GC consumptions in each paired-durations of six and 12 months before and after anti-TNF therapy revealed significantly decreased mean daily GC intake. Achieved reduction rates were $11 \%$ at six months and $30 \%$ at 12 months. Initiation of anti-TNF therapy was found to be associated with reduced NSAID use. This pattern was similar as that of GC use except for consumption comparisons in the first episode.

Conclusion: Anti-TNF treatment reduced NSAID and GC usage in patients with RA. Evidence of decreased consumption for both medication groups have been reached statistical significance after the first six months of biologic treatment initiation.

Keywords: Corticosteroid sparing; glucocorticoid; nonsteroidal anti-inflammatory drug; tumor necrosis factor inhibitor; rheumatoid arthritis.
\end{abstract}

Anti-tumor necrosis factor (anti-TNF) agents have shown substantial efficacy in controlling activity and preventing disease progression of rheumatoid arthritis (RA). ${ }^{1}$ TNF blocking agents are indicated for treatment of severely active RA patients who have had an inadequate response to conventional therapy including glucocorticoids (GCs) and nonsteroidal anti-inflammatory drugs (NSAIDs).

Despite introduction of biologic agents, GCs and NSAIDs are still widely prescribed co-therapies by rheumatologists for the treatment of RA. GC therapy can also reduce destructive erosions of RA while improving symptoms. ${ }^{2-7}$ However, GCs have several potential side effects, particularly in prolonged use and high doses.
Thus, tapering GC doses down to a minimal intake and minimizing long-term use of GCs remain the major challenge to balance benefits and risks of side effects including hypertension, weight gain, osteoporosis, fractures, osteonecrosis, increased cardiovascular risk, peptic ulcer disease, and infection. ${ }^{8-16}$

Nonsteroidal anti-inflammatory drugs are the least potent drugs used for RA. These drugs relieve pain by reducing inflammation, but they do not affect the course of the disease. ${ }^{17}$ As the most commonly prescribed pain relievers, they aim to facilitate living with RA. However, NSAIDs also increase the risk of peptic ulcers and gastrointestinal bleeding and also have adverse 
cardiovascular effects. ${ }^{18-20}$ Reducing exposure to these co-therapies is desirable to decrease the incidence of therapy-related adverse outcomes in RA patients.

Unlike disease-modifying antirheumatic drugs (DMARDs), which can take up to 8-12 weeks to work, biologic agents tend to work as quick as two weeks. Although anti-TNF therapy is effective in controlling disease activity, only a limited number of studies with small sample sizes have addressed their effects on the use of GCs and NSAIDs. ${ }^{21-24}$ Effective treatment modalities should not only control disease activity but also reduce the need for co-therapies such as GCs, and NSAIDs. Currently, limited information is available concerning the potential GC sparingeffect of anti-TNF therapy in RA in terms of reducing the need for these co-therapies.

In this retrospective study, we aimed to investigate whether use of NSAIDs or GCs decreases over different time intervals before and after anti-TNF therapy in RA patients.

\section{PATIENTS AND METHODS}

Data were obtained from the electronic prescription records of the health assistance branch of the Pension Fund of Turkish Republic, also known as "Emekli Sandığı", which was part of the Social Security Institution in Turkey. This national institution covered approximately $21 \%$ of Turkish population in years 2000 to 2005 .

A query was conducted using electronic prescription database of the Pension Fund of Turkey from 2000 to 2005 to detect patients aged 18 years or older who were administered anti-TNF agents. Anti-TNF inhibitors including only infliximab, etanercept and adalimumab were available in Turkish market at that time; therefore, those agents were included in the study.

The database contained 469 patients who had been prescribed anti-TNF therapy. Of these patients, 424 (90\%) were diagnosed as having RA on the basis of health status reports (95 males, 329 females; mean age $52.5 \pm 13.3$ years; range 18 to 80 years).

All prescription records of the patients registered as RA were visible in the system.
However, since validation of diagnosis to ascertain fulfillment of the 1987 revised American College of Rheumatology criteria for RA was not possible due to database limitations, reported diagnoses were accepted to be eligible.

Patient information including demographic data, name of institutions where the TNF inhibitors were prescribed and diagnoses as well as all prescription records were extracted from the database excluding patient identity details, and a new database was created for further analysis.

All NSAIDs (only oral forms) and GCs (oral+parenteral forms) which were available on Turkish pharmaceutical market during those years were screened in the electronic patient database. All parenteral forms of GCs were accepted to be used through parenteral route, as it was not possible to differentiate intra-articular administration of parenteral forms from the records.

The study protocol was reviewed and approved by the local ethics committee and the drug regulatory authorities. In the first stage, patients were categorized into three groups according to the duration of continuous antiTNF agent utilization in the defined period (Episode-1: >90 days, Episode-2: >180 days, Episode-3: $>365$ days). In the second stage, NSAID and GC consumptions ( $\geq 1$ prescription) of RA patients in the preceding three, six and 12 months before the initiation of first biologic agent were compared with same duration of consumptions after the initiation of biologic agent in each paired time intervals respectively (Figure 1).

The first recognized biologic agent in the prescription records was accepted as "index prescription" and its date was noted as start date of the medication. Biologic treatment duration was calculated from total prescribed biologic units individually for each. We assessed the duration of treatment based on worldwide registered doses of TNF inhibitors: etanercept at $25 \mathrm{mg}$ twice a week, infliximab at $3 \mathrm{mg} / \mathrm{kg}$ by average adult body weight $(70 \mathrm{~kg}$ ) (initial loading dose at week zero, two and six, and thereafter every eight weeks), and adalimumab at $40 \mathrm{mg}$ every two weeks. By this way, prescription data were transformed into the certain episodes 


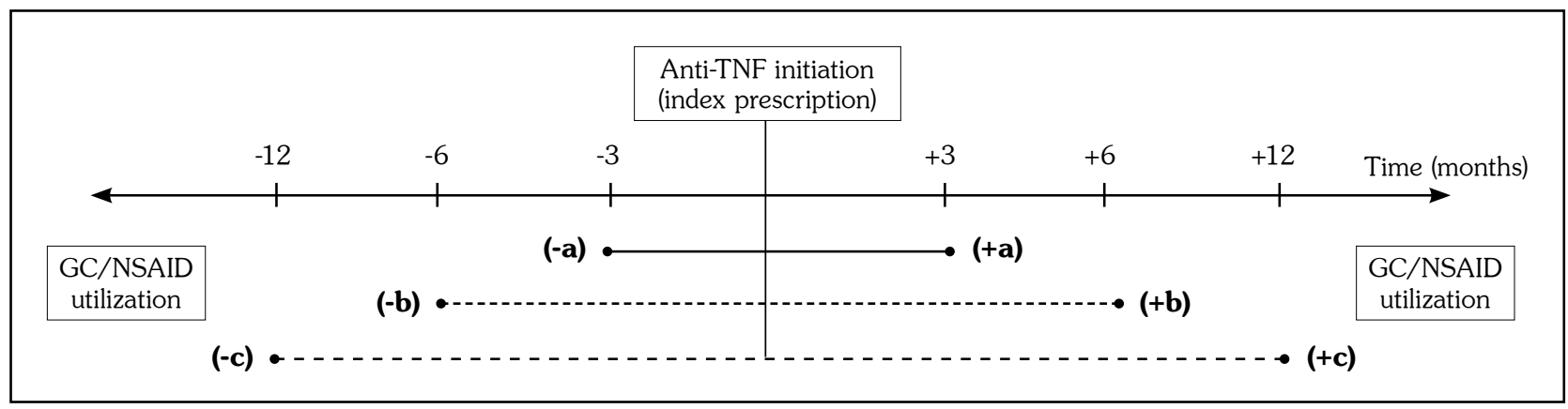

Figure 1. Schematic comparison of drug utilization according to different time intervals. NSAID and GC consumptions $(\geq 1$ prescription) analyzed within each paired time intervals [(-a) with $(+a),(-b)$ with $(+b)$ and $(-c)$ with $(+c)]$ in RA patients treated with sustained anti-TNF therapy according to definition of episode-1, 2 and 3. TNF: Tumor necrosis factor; GC: Glucocorticoid; NSAID: Nonsteroidal anti-inflammatory drug.

of drug use. As this study did not aim to evaluate the impact of switching between antiTNF regimens on concomitant medication, we only calculated total biologic treatment duration from the aforementioned formula, regardless of the particular anti-TNF agent.

Glucocorticoid drug utilizations were all converted to the "prednisone equivalent dose" and NSAID drug utilizations were calculated using the defined daily dose system. Defined daily dose is a statistical measure of drug consumption, defined by the World Health Organization. ${ }^{25}$ This enables the total volume of prescriptions for each medication to be converted into a standardized daily drug dose. Aggregation of all the defined daily doses for a group of related drugs can provide a measure of the notional days of treatment for a therapeutic drug group. A brief explanation of the study design was presented in Figure 1.

\section{Statistical analysis}

Patients' characteristics were reported as number (percentage) for categorical variables and mean \pm standard deviation for continuous variables. To search for a GC or a NSAID sparing effect of anti-TNF initiation, paired comparisons were performed between GC and NSAID intake before and after index prescription, using Wilcoxon test for quantitative variables. For all statistical analyses, a p-value less than 0.05 was considered statistically significant. Statistical analyses involved use of the SPSS version 16.0 statistical software package (SPSS Inc., Chicago, IL, USA).

\section{RESULTS}

Our major findings with $424 \mathrm{RA}$ patients are as follows: (i) the most commonly prescribed antiTNF medications were 202 infliximab (48\%), 195 etanercept (46\%), and 27 adalimumab (6\%); (ii) delayed entrance of adalimumab into the Turkish market resulted in relatively lower prescription rates; (iii) biological treatment was discontinued after the first prescription in 32 patients (6\%) (13 infliximab, 15 etanercept, four adalimumab).

Number of RA patients and their drug usage characteristics within each paired-time intervals before and after initiation of biologic therapy related with the duration of anti-TNF agent utilization were summarized in Table 1.

The percentage of patients using NSAIDs was always higher than the percentage of patients using GCs in all treatment groups (Table 1). After initiation of anti-TNF treatment, number of patients using GC decreased in each paireddurations of three, six and 12 months and the reduction rates were $11.9 \%, 10.4 \%$ and $15.3 \%$, respectively. Similarly, reductions by $10.6 \%$, $10.3 \%$ and $11.0 \%$ were observed in the number of patients using NSAIDs (Table 1).

Only in episode-1 ( $>90$ days of anti-TNF), percentage of patients increasing GC dosage (35\%) was higher than percentage of patients decreasing GC dosage (32\%). However, for NSAID consumption, the proportion of patients who needed to decrease the dosage (41\%) was higher than the patients who needed to increase the dosage (34\%). In the other episodes of treatment groups, RA patients showed similar 


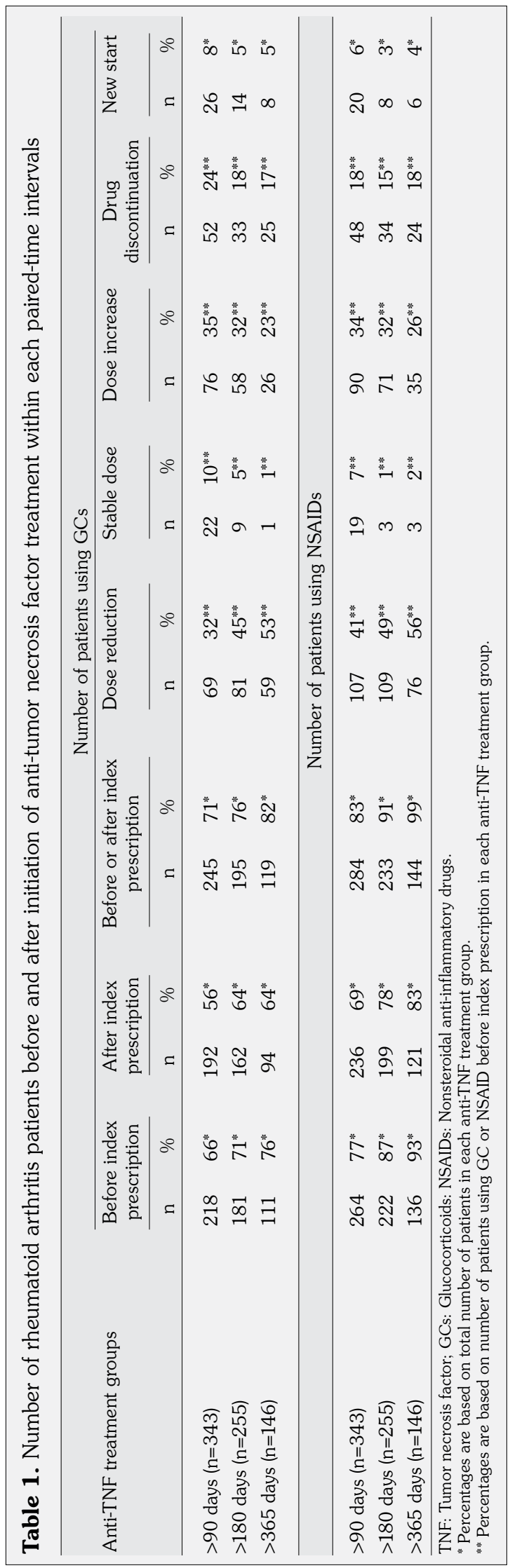

evidence of clinical benefit with higher rates of patients reducing the dosage for both drugs after initiation of anti-TNF therapy. The ratios for "dose reduction" to "dose increase" for each treatment group were $0.91,1.40$ and 2.27 for GC consumption and 1.19, 1.54 and 2.17 for NSAID consumption, respectively. Ratios for "drug discontinuation" to "new start" for each treatment group were 2, 2.36 and 3.12 for GC consumption and $2.4,4.25$ and 4 for NSAID consumption, respectively (Table 1).

The relationship between NSAID and GC use was analyzed for each anti-TNF treatment group. Mean daily use of NSAID and GC within each paired-time intervals $[(-a)$ with $(+a),(-b)$ with $(+b)$ and $(-c)$ with $(+c)$ ] before and after anti-TNF therapy was summarized in Table 2.

In episode-1, the mean daily GC doses before $(7.5 \pm 6.9 \mathrm{mg} /$ day $)$ and after anti-TNF initiation $(7.4 \pm 7.0 \mathrm{mg} / \mathrm{day})$ indicate only $1 \%$ reduction in GC consumption which is not significant $(p=0.077)$.

In episode-2 ( $>180$ days of anti-TNF), the mean daily GC doses before $(5.8 \pm 4.8 \mathrm{mg} / \mathrm{day})$ and after anti-TNF initiation $(5.1 \pm 5.1 \mathrm{mg} /$ day $)$ indicate an $11 \%$ reduction in GC consumption. The treatment difference was statistically significant $(p=0.001)$.

In episode-3 (>365 days of anti-TNF), the mean daily GC doses before $(4.7 \pm 3.9 \mathrm{mg}$ /day) and after anti-TNF initiation $(3.3 \pm 3.5 \mathrm{mg} /$ day $)$ indicate a $30 \%$ reduction in GC consumption with a significant difference $(p=0.000)$.

Mean daily NSAID use before and after antiTNF therapy increased $13 \%$ in the first group of patients which was not significant $(p=0.078)$. However, in the second and third group of patients, NSAID use decreased 10\% and 25\% respectively with a significant difference $(p<0.001$, $\mathrm{p}<0.001$, respectively).

\section{DISCUSSION}

Our study showed a significantly decreased mean daily GC intake in comparison with GC consumption in each paired-durations (6-12 months) of pre-and post-anti-TNF initiation and the reduction rates were $11 \%$ and $30 \%$ respectively. Insignificantly decreased GC use was observed 
Table 2. Summary of glucocorticoid and nonsteroidal anti-inflammatory drug consumption and dose analysis in certain time intervals before and after start of anti-tumor necrosis factor treatment

\begin{tabular}{|c|c|c|c|c|c|c|c|}
\hline \multirow[t]{2}{*}{ Medication } & \multirow[t]{2}{*}{$\begin{array}{l}\text { Duration } \\
\text { (months) }\end{array}$} & \multicolumn{2}{|c|}{$\begin{array}{l}\text { Before index } \\
\text { prescription }\end{array}$} & \multicolumn{2}{|c|}{$\begin{array}{l}\text { After index } \\
\text { prescription }\end{array}$} & \multirow{2}{*}{$\begin{array}{c}\begin{array}{c}\text { Increase or } \\
\text { decrease rate }\end{array} \\
\%\end{array}$} & \multirow{2}{*}{$\frac{\text { Significance }}{}$} \\
\hline & & Mean \pm SD & Range & Mean \pm SD & Range & & \\
\hline \multirow[t]{3}{*}{ Mean daily GC dose (mg/day)* } & 3 & $7.5 \pm 6.9$ & $0.56-36.67$ & $7.4 \pm 7.0$ & $0.56-45.56$ & 1.0 & 0.077 \\
\hline & 6 & $5.8 \pm 4.8$ & $0.28-30.56$ & $5.1 \pm 5.1$ & $0.28-31.94$ & 11.1 & 0.001 \\
\hline & 12 & $4.7 \pm 3.9$ & $0.16-25.34$ & $3.3 \pm 3.5$ & $0.14-22.9$ & 30.2 & 0.000 \\
\hline \multirow[t]{3}{*}{ Mean daily NSAID dose $e^{* * *}$} & 3 & $1.0 \pm 1.8$ & $0.07-14.67$ & $1.1 \pm 2.0$ & $0.07-19.56$ & $13.4^{* * *}$ & 0.078 \\
\hline & 6 & $0.9 \pm 2.0$ & $0.06-19.56$ & $0.9 \pm 1.5$ & $0.04-15.89$ & 9.6 & 0.000 \\
\hline & 12 & $0.9 \pm 2.0$ & $0.05-15.16$ & $0.7 \pm 1.4$ & $0.02-11.45$ & 24.7 & 0.000 \\
\hline
\end{tabular}

SD: Standard deviation; GC: Glucocorticoid; NSAID: Nonsteroidal anti-inflammatory drug; P values of 0.000 mean that $\mathrm{p}<0.0001$; * Per patient-day prednisone equivalent; "* Per patient-day defined daily dose equivalent; ${ }^{* * *}$ Increased NSAID use.

in the first three months of anti-TNF initiation; however, statistical significance emerged over the first 6-12 months of therapy. Thus, a three-month observation period appears to be short to observe a change in GC use.

However, some studies in the literature revealed a significant decrease in GC use as early as in the first three months of treatment. ${ }^{21-24}$ It is difficult to explain the slow onset of action in our study which may be related to the use of co-therapies including other non-biologic DMARDs and NSAIDs. On the other hand, NSAID utilization, even for a short period of time, has been shown to increase over the first three months of anti-TNF initiation. Despite their benefits, many patients are reluctant to use systemic GCs due to fear from systemic side-effects and the common misconceptions that exist regarding the safety of these agents. Initially, awareness of potential side-effects on GCs may lead some patients to raise their NSAID dosing in order to balance themselves against additional risks associated with TNF inhibitors that are new to the market.

In contrast to studies evaluating anti-TNF inhibitors, no reduction in prednisolone dosage was possible in the patients treated with nonbiologic DMARD (leflunomide) even at 12 months of treatment. ${ }^{21,24}$

In the present study, a mean of $7.5 \mathrm{mg} /$ day of prednisone equivalent dose has been shown to be used just before anti-TNF initiation for RA patients. At the time of anti-TNF initiation, this amount of GC dose is similar with the previously published studies with mean daily dose of $8.2 \mathrm{mg}$ and $7.5 \mathrm{mg} \cdot{ }^{21-23}$ According to the prescription records, clinicians advocated low doses of GCs to RA patients even in clinically active disease while waiting results of conventional DMARD therapy.

In our study, anti-TNF agents conferred significant advantage in reducing mean daily GC use which suggested that improved disease control may lead to decreased need for GCs. This magnitude of GC sparing (30\%) comparing mean daily GC intake in the year before to that in the year after anti-TNF initiation is similar with a previously published retrospective clinical trial of GC usage (38\%). ${ }^{22}$

In another study, GC sparing effect of a newly started DMARD (classic + biologic) regimen has not been verified in terms of mean daily GC use in RA patients at six to 12 months. ${ }^{24}$ The study assembled four retrospective cohorts of RA patients, and the proportion of patients initiated an anti-TNF agent ranged from 25 to $30 \%$ in all the cohorts. Nonetheless, there was a decrease in their overall use of GCs and NSAIDs after DMARD (conventional or biologics) initiation. More patients stopped using GCs and NSAIDs compared to those who started them, which resulted in an $8.9 \%$ and $12.9 \%$ decrease in the overall use of GCs and NSAIDs respectively. ${ }^{24}$ Detailed proportions of our RA patients before and after initiation of anti-TNF treatment within each paired-time intervals were summarized in Table 1.

According to the recent data, a significant GC-sparing effect of anti-TNF agents have ability to reduce GC use in clinical practice. 
This is an important finding, as steroid toxicity is common and often leads to significant comorbidity such as Cushingoid features, weight gain, mood disorders, glucose intolerance, osteoporosis, and increased susceptibility to infection and cardiovascular disease. ${ }^{26}$ The prednisolone equivalent dosage was decreased during the first 12 months after anti-TNF initiation (Table 2).

As we were unable to measure disease activity before and after treatment due to database limitations, other reasons for decreased GC use should be considered such as improved disease control, or physician's or patient's desire to limit adverse effects.

Tumor necrosis factor inhibitor initiation is associated with reduced NSAID use along with decreased corticosteroid use. We showed a similar pattern for NSAID use. Nonetheless, statistically insignificantly increased NSAID use (13\%) was observed in the first three months of anti-TNF initiation compared with the previous three months of NSAID use before index prescription. However, comparison of consumptions in each paired-durations (six and 12 months) of pre- and post- anti-TNF initiation revealed statistically significant reductions by $10 \%$ and $25 \%$ respectively.

Limited number of studies have examined the effects of anti-TNF initiation on NSAID use. ${ }^{23,24}$ To the best of our knowledge, this is the first study NSAID utilization was measured and compared as the total number of defined daily doses. Naumann et al. ${ }^{23}$ reported that the proportion of NSAID users decreased by $16 \%$ (from $75 \%$ to $59 \%$ ) after a year of anti-TNF initiation. In another study, overall reduction in the proportion of NSAID users was shown to be $13 \%$ after the same period of time with anti-TNF therapy. ${ }^{24}$ Direct comparison of the results of our study with these two studies is not possible due to methodological differences. These two studies did not analyze the existence of any reduction in the average daily GC or NSAID utilization among the patients who started anti-TNF drugs. Instead, overall reduction in the proportion of NSAID users was demonstrated. Nevertheless, all of these studies including current study highlight an apparent NSAID-sparing effect of anti-TNF initiation.
Although remarkable consistency in findings across diverse studies suggests that improved disease control due to TNF inhibitor initiation may decrease NSAID use, other reasons for decreased use cannot be excluded. For instance, information on alternate indications for those co-therapies like leflunomide and/or methotrexate were not available. In the same way, decreased NSAID utilization can be related to the recognition of gastrointestinal and cardiovascular toxicity.

Our study has some limitations: (i) We ascertained medication use by calculating prescription data but we were unable to measure absolute adherence to therapies; (ii) the proportion of identified patients who met American College of Rheumatology criteria for $\mathrm{RA}$ is unknown. Any residual misclassification could be available in the database, nonetheless similar disadvantages is present in almost every database; (iii) the presence of other comorbidities might also be the reason for lower GC and NSAID use in our database; (iv) a patient can receive almost any medication without a prescription or medical record. Indeed, NSAIDs and GCs are not expensive in our pharmaceutical market, and purchasing medications directly from pharmacies is somewhat convenient to patients without any requirement for a prescription; $(v)$ finally, there was no control group of patients undergoing DMARD therapy.

The response observed in randomized controlled trials (RCTs) can be different from the response achieved in daily clinical practice. $\mathrm{RCT}$ do not necessarily reflect real life patient populations that are exposed to drug therapy in clinical practice due to specified protocol requirements. Therefore, data from clinical trials are less useful compared to real life data. In RCTs, GCs daily dose often has to be stable during the whole study period; therefore, results of RCTs cannot entirely address the question of GC tapering. Our data are complementary to results from randomized controlled clinical trials due to the external validity of such trials.

In conclusion, anti-TNF treatment was shown to reduce NSAID and GC usage in patients with RA. Statistically significantly decreased requirement of NSAIDs and GCs has been reached in the first six months after biologic 
medication was commenced. The results of our study suggest that treatment with anti-TNFs during the first six months in RA patients in a real-life setting helps to reduce GC intake in patients receiving low doses of GCs.

\section{Declaration of conflicting interests}

The authors declared no conflicts of interest with respect to the authorship and/or publication of this article.

\section{Funding}

The authors received no financial support for the research and/or authorship of this article.

\section{REFERENCES}

1. Donahue KE, Gartlehner G, Jonas DE, Lux LJ, Thieda $\mathrm{P}$, Jonas $\mathrm{BL}$, et al. Systematic review: comparative effectiveness and harms of disease-modifying medications for rheumatoid arthritis. Ann Intern Med 2008;148:124-34.

2. A COMPARISON of cortisone and aspirin in the treatment of early cases of rheumatoid arthritis; a report by the Joint Committee of the Medical Research Council and Nuffield Foundation on Clinical Trials of Cortisone, A.C.T.H., and Other Therapeutic Measures in Chronic Rheumatic Diseases. Br Med J 1954;1:1223-7.

3. EMPIRE Rheumatism Council: multi-centre controlled trial comparing cortisone acetate and acetyl salicylic acid in the long-term treatment of rheumatoid arthritis; results of three years' treatment. Ann Rheum Dis 1957; 16:277-89.

4. A COMPARISON of prednisolone with aspirin on other analgesics in the treatment of rheumatold rthritis. Ann Rheum Dis 1959;18:173-88.

5. Harris ED Jr, Emkey RD, Nichols JE, Newberg A. Low dose prednisone therapy in rheumatoid arthritis: a double blind study. J Rheumatol 1983;10:713-21.

6. Kirwan JR. The effect of glucocorticoids on joint destruction in rheumatoid arthritis. The Arthritis and Rheumatism Council Low-Dose Glucocorticoid Study Group. N Engl J Med 1995;333:142-6.

7. van Everdingen AA, Jacobs JW, Siewertsz Van Reesema $\mathrm{DR}$, Bijlsma JW. Low-dose prednisone therapy for patients with early active rheumatoid arthritis: clinical efficacy, disease-modifying properties, and side effects: a randomized, double-blind, placebo-controlled clinical trial. Ann Intern Med 2002;136:1-12.

8. Pirpiris M, Sudhir K, Yeung S, Jennings G, Whitworth JA. Pressor responsiveness in corticosteroid-induced hypertension in humans. Hypertension 1992;19:567-74.

9. Connell JM, Whitworth JA, Davies DL, Lever AF, Richards AM, Fraser R. Effects of ACTH and cortisol administration on blood pressure, electrolyte metabolism, atrial natriuretic peptide and renal function in normal man. J Hypertens 1987;5:425-33.

10. Reid IR. Pathogenesis and treatment of steroid osteoporosis. Clin Endocrinol (Oxf) 1989;30:83-103.

11. van Staa TP, Leufkens HG, Cooper C. The epidemiology of corticosteroid-induced osteoporosis: a meta-analysis. Osteoporos Int 2002;13:777-87.

12. Wei L, MacDonald TM, Walker BR. Taking glucocorticoids by prescription is associated with subsequent cardiovascular disease. Ann Intern Med 2004;141:764-70.

13. Dixon WG, Suissa S, Hudson M. The association between systemic glucocorticoid therapy and the risk of infection in patients with rheumatoid arthritis: systematic review and meta-analyses. Arthritis Res Ther 2011;13:R139.

14. Messer J, Reitman D, Sacks HS, Smith H Jr, Chalmers TC. Association of adrenocorticosteroid therapy and peptic-ulcer disease. N Engl J Med 1983;309:21-4.

15. Kyle V, Hazleman BL. Treatment of polymyalgia rheumatica and giant cell arteritis. II. Relation between steroid dose and steroid associated side effects. Ann Rheum Dis 1989;48:662-6.

16. Mankin HJ. Nontraumatic necrosis of bone (osteonecrosis). N Engl J Med 1992;326:1473-9.

17. American College of Rheumatology Subcommittee on Rheumatoid Arthritis Guidelines. Guidelines for the management of rheumatoid arthritis: 2002 update. Arthritis Rheum 2002;46:328-46.

18. Armstrong EP, Malone DC. The impact of nonsteroidal anti-inflammatory drugs on blood pressure, with an emphasis on newer agents. Clin Ther 2003;25:1-18.

19. Huang JQ, Sridhar S, Hunt RH. Role of Helicobacter pylori infection and non-steroidal anti-inflammatory drugs in peptic-ulcer disease: a meta-analysis. Lancet 2002;359:14-22.

20. Lanza FL. Endoscopic studies of gastric and duodenal injury after the use of ibuprofen, aspirin, and other nonsteroidal anti-inflammatory agents. Am J Med 1984;77:19-24.

21. Geborek P, Crnkic M, Petersson IF, Saxne T. Etanercept, infliximab, and leflunomide in established rheumatoid arthritis: clinical experience using a structured follow up programme in southern Sweden. Ann Rheum Dis 2002;61:793-8.

22.Seror R, Dougados M, Gossec L. Glucocorticoid sparing effect of tumour necrosis factor alpha inhibitors in rheumatoid arthritis in real life practice. Clin Exp Rheumatol 2009;27:807-13.

23. Naumann L, Huscher D, Detert J, Spengler M, Burmester GR, Buttgereit F. Anti-tumour necrosis factor \{alpha\} therapy in patients with rheumatoid arthritis results in a significant and long-lasting decrease of concomitant glucocorticoid treatment. Ann Rheum Dis 2009;68:1934-6. 
24. Kawai VK, Grijalva CG, Arbogast PG, Curtis JR, Solomon DH, Delzell E, et al. Changes in cotherapies after initiation of disease-modifying antirheumatic drug therapy in patients with rheumatoid arthritis. Arthritis
Care Res (Hoboken) 2011;63:1415-24.

25. Available from: www.whocc.no/atcddd/

26. Rosen J, Miner JN. The search for safer glucocorticoid receptor ligands. Endocr Rev 2005;26:452-64. 